\title{
Topological cathodes: Controlling the space charge limit of electron emission using metamaterials
}

\author{
David H. Dowell@* \\ SLAC National Accelerator Laboratory, 2575 Sand Hill Road, Menlo Park, California 94025, USA
}

(Received 20 February 2019; published 23 August 2019)

\begin{abstract}
The space charge limit (SCL) of emission from photocathodes sets an upper limit on the performance of both high- and low-field electron guns. Generally, one is forced to strike a compromise between the space charge limit and the cathode's intrinsic emittance [I. Bazarov et al., Phys. Rev. Lett. 102, 104801 (2009)]. However, it is possible to nearly eliminate the SCL due to the image charge by engineering the topography of the cathode's surface. A cathode with a surface plasma frequency below the frequency spectrum of the accelerating electrons will greatly reduce the polarization of the cathode and its image-charge field, thereby mitigating the cathode's space charge limit for photoelectric, thermionic, and field emission. In the work presented here, a theory for the image-charge field of a disk of charge being accelerated from the cathode surface is developed which includes the frequency-dependent effects of the cathode's dielectric function. This theory verifies the concept of SCL mitigation with a wire array metamaterial to design a metacathode with greatly reduced image-charge fields. The details of the holistic metacathode design spanning 6 orders of magnitude in scale are described.
\end{abstract}

DOI: 10.1103/PhysRevAccelBeams.22.084201

\section{INTRODUCTION}

Our ability to assemble subwavelength, atomlike structures into manmade metamaterials with exotic properties is revolutionizing the fields of photonics, acoustics, and the material sciences. Metamaterials consisting of 3D arrays of "artificial atoms" in the form of miniature resonators have been shown to exhibit unnatural optical properties like a negative index of refraction. Research of these new materials is beginning to make even outlandish concepts like optical cloaking and perfect lenses demonstrable possibilities.

The goal of this paper is to incorporate these new metamaterial ideas into beam physics and accelerator technology. Here, these concepts are applied to design a cathode with the goal of mitigating the cathode's space charge limit of emission. This is done by choosing a metasurface whose plasma frequency is below the frequency spectrum of the emitted electrons' image-charge field. It is shown that such a metasurface can greatly reduce the image-charge field and thereby mitigate the space charge limit (SCL).

A low image-charge field would enable all guns to operate with higher-density bunches. A small image-charge field would allow using a smaller laser spot without concern of the

*dowell@slac.stanford.edu; ddowell@ centurylink.net

Published by the American Physical Society under the terms of the Creative Commons Attribution 4.0 International license. Further distribution of this work must maintain attribution to the author(s) and the published article's title, journal citation, and DOI. space charge limit, thereby reducing the beam's mean transverse energy and intrinsic emittance. A smaller initial beam size would also reduce the chromatic and geometric aberrations in the injector's optics. In addition, bunch length elongation and other longitudinal dynamics should become more linear without the image field.

In this work, the image method is used to obtain the image-charge field near the surface of the cathode. This field is evaluated at the electron bunch location and travels with the bunch as it accelerates from the cathode. Fourier transforms are used to combine the image-charge field produced by the bunch with the cathode's surface loss function to calculate the total, frequency-dependent electric field at the bunch. A simple wire array metamaterial is used with the theory to calculate the range of the time-dependent fields generated during the bunch-cathode interaction.

Here, we ignore the space-charge forces between electrons within the bunch. These forces result from the bunch's self-energy or potential energy of assembly which drives radial and longitudinal expansion. Researchers have shown in both theory [1,2] and experiment [3] that, if this expansion is linear, then it produces no emittance growth.

Section II describes the connection between the image charge and the dielectric function. The relationship between the dielectric function and the surface loss function (also known as the image-to-real charge ratio) is given, and the conditions for controlling the SCL are described. Section III develops a thin disk model of the beam which combines the time dependence of the image field with the surface response function. In Sec. IV, 
a metasurface consisting of a rectangular array of parallel wires is used to illustrate the large range of dielectric properties a metamaterial can produce, and the design features needed for a practical metacathode are discussed. The section ends with a brief review of the nanotechnology relevant to fabricating the metacathode. Section V describes how these capabilities are factored into the metacathode design. Section VI describes the metasurface features and discusses its design details. Section VII contains concluding remarks with suggestions for further research.

\section{THE IMAGE CHARGE AND THE DIELECTRIC FUNCTION OF CATHODES}

The mirror image method [4] gives the ratio of the image charge $q^{\prime}$ to the real external charge $q$ as

$$
\frac{q^{\prime}}{q}=-\left(\frac{\epsilon_{c}-1}{\epsilon_{c}+1}\right),
$$

where $\epsilon_{c}$ is the cathode's relative electric permittivity defined in terms of its electric permittivity $\epsilon_{\text {cathode }}$ and electric susceptibility $\chi_{e}$ :

$$
\epsilon_{\text {cathode }}=\epsilon_{0} \epsilon_{c}=\epsilon_{0}\left(1+\chi_{e}\right) .
$$

In this paper, the frequency-dependent relative permittivity $\epsilon_{c}(\omega)$ is referred to as the dielectric function of the cathode.

The surface loss function at low momentum transfer [5] is

$$
g(q \rightarrow 0, \omega)=\left(\frac{\epsilon(\omega)-1}{\epsilon(\omega)+1}\right) .
$$

Comparing with Eq. (1) indicates the image charge depends upon the frequency and is the negative of the surface loss function when $q \sim 0$ for photoemission. Thus, we define the surface loss function for the cathode as

$$
g_{c}(\omega) \equiv\left(\frac{\epsilon_{c}(\omega)-1}{\epsilon_{c}(\omega)+1}\right)=-\frac{q^{\prime}}{q} .
$$

The negative of the image-to-real charge ratio is the cathode's surface loss function.

The Drude theory of metals [6] defines the dielectric function in terms of the metal's plasma frequency and relaxation or collision time. In this theory, the dielectric function is specified by two parameters, $\omega_{p}$ and $\tau_{p}$ :

$$
\epsilon(\omega)=1-\frac{\omega_{p}^{2}}{\omega\left(\omega+\frac{i}{\tau_{p}}\right)} .
$$

Here, $\omega_{p}$ is the plasma frequency which is related to $n$, the number density of conduction band electrons,

$$
\omega_{p}^{2}=\frac{n e^{2}}{\epsilon_{0} m}
$$

and the relaxation time $\tau_{p}$ depends upon both the conduction band density and the de electrical conductivity $\sigma$ :

$$
\tau_{p}=\frac{m \sigma}{n e^{2}},
$$

where $m$ is the mass of an electron and $e$ is its charge. These two properties determine the magnitude and frequency behavior of $q^{\prime} / q$ and the SCL.

Using the Drude dielectric function in Eq. (4) allows expressing the cathode's surface response function in terms of these two fundamental quantities:

$$
g_{c}(\omega)=\frac{\omega_{p}^{2}}{\omega_{p}^{2}-2 \omega\left(\omega+\frac{i}{\tau_{p}}\right)} .
$$

Table I gives the free electron properties of copper, a material commonly used as a cathode in high-field rf guns. For metals, the plasma frequency is 4 orders of magnitude higher than the high-frequency edge of the image-charge field's frequency spectrum. Since the bunch time scale is of the order of picoseconds, the high-frequency edge of the image field is of the order of $10^{12} \mathrm{rad} / \mathrm{s}$. The table shows that the plasma frequency of copper is $1.64 \times 10^{16} \mathrm{rad} / \mathrm{s}$. Therefore, for metal cathodes, one always has $\omega \ll \omega_{p}$, $g_{c}(\omega \approx 0)=1$, and $q^{\prime}=-q$.

In the case of multialkali cathodes [7], the imaginary part of the dielectric function tends toward zero and $\operatorname{Re}\left(\epsilon_{c}\right) \approx 6$, which gives $g_{c} \approx 1$ for frequencies at and lower than $\omega_{e}$. So again $q^{\prime}=-q$ at the image-field frequencies. Therefore, both metal and multialkali photocathodes have the same negative image-charge fields.

The Drude dielectric function given in Eq. (5) is a complex function whose real and imaginary parts are plotted in Fig. 1. The figure shows the imaginary part diverging at frequencies below the plasma frequency, whereas the real part becomes the dc conductivity at $\omega=0$.

The ratio of the image charge to the real charge [or $\left.-g_{c}(\omega)\right]$ for copper is shown in Fig. 2. The calculations use Eq. (5) for the cathode dielectric function with copper's plasma frequency and relaxation time. Both the real and imaginary parts diverge at a transition frequency where the

TABLE I. Free electron properties of copper. The electron densities are taken from Table 1.1 and the relaxation times from Table 1.3 in Ref. [6].

\begin{tabular}{lc}
\hline \hline Number density $n\left(/ \mathrm{m}^{3}\right)$ & $8.47 \times 10^{28}$ \\
Relaxation time $\tau(\mathrm{s})$ & $2.7 \times 10^{-14}$ \\
Plasma freq. $\omega_{p}(\mathrm{rad} / \mathrm{s})$ & $1.64 \times 10^{16}$ \\
dc conductivity $(\mathrm{S} / \mathrm{m})$ & $6.3 \times 10^{7}$ \\
Transition freq. $\omega_{t}(\mathrm{rad} / \mathrm{s})$ & $1.16 \times 10^{16}$ \\
\hline \hline
\end{tabular}



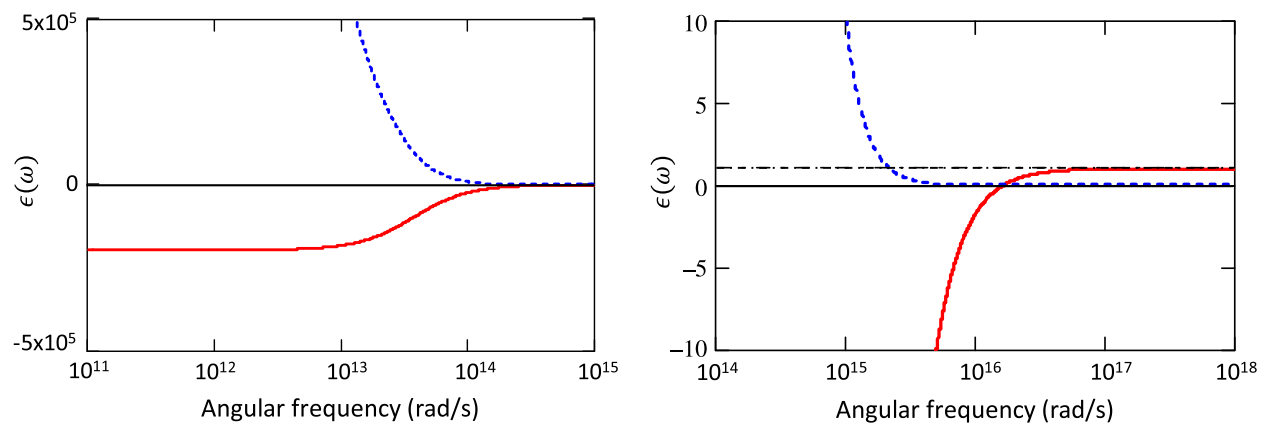

FIG. 1. The complex dielectric function for copper at room temperature with different vertical and horizontal scales. The real part is plotted with the solid red curves, and the imaginary part is plotted using dashed blue curves. The plasma frequency is $1.64 \times 10^{16} \mathrm{rad} / \mathrm{s}$ and is the frequency where the real part is zero.

real part switches between -1 to 0 and where the imaginary part has a narrow, negative peak. This is the frequency where the cathode's image charge transitions from that of a metal $\left(q^{\prime}=-q\right)$ to that of a vacuum $\left(q^{\prime}=0\right)$. The vacuumlike behavior is identical to the well-known phenomenon of ultraviolet transparency in metals [8]. In the present case, transparency occurs when $q^{\prime}=0$ and, from the beam's perspective, the cathode "disappears."

The transition between metal-like and vacuumlike occurs when

$$
\operatorname{Re}\left[g_{c}\left(\omega_{t}\right)\right]=\frac{1}{2}
$$

where $\omega_{t}$ is the transition frequency. Using the Drude dielectric function in the cathode's response function in Eq. (9) and solving for $\omega_{t}$ gives

$$
\omega_{t}^{2}=\frac{1}{2 \tau_{p}^{2}} \sqrt{1+\tau_{p}^{4} \omega_{p}^{4}}-\frac{1}{2 \tau_{p}^{2}} .
$$

Usually, for metals like copper, the plasma frequency is greater than $\frac{1}{\tau_{p}}$, and the transition frequency can be approximated by

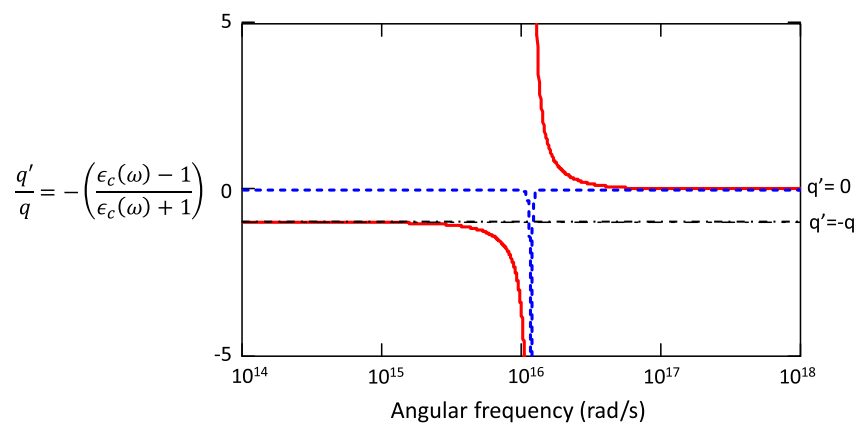

FIG. 2. The image-charge to real-charge ratio $q^{\prime} / q=-g_{c}(\omega)$, as a function of the frequency for copper. The dashed blue curve is the imaginary part, and the solid red curve is the real part. A dash-dot line is drawn at $q^{\prime}=-q$ assumed in most current cathode models. The transition frequency for copper is $\omega_{t}=$ $1.16 \times 10^{16} \mathrm{rad} / \mathrm{s}$.

$$
\omega_{t} \cong \frac{\omega_{p}}{\sqrt{2}} \text { when } \quad \frac{1}{\tau_{p}} \ll \omega_{p} .
$$

On the other hand, a metasurface can be designed to have a very short relaxation time in order to damp the $\omega_{t}$ oscillations. In this case, $\tau_{p}^{4} \omega_{p}^{4} \ll 1$, and the transition frequency becomes the difference of two large numbers as can be seen in Eq. (10). This numerical difficulty can be removed by expanding the square root in powers of $\tau_{p}$. The first term of this series cancels the $-\frac{1}{2 \tau_{p}^{2}}$ term, leaving

$\omega_{t}^{2} \cong \frac{\omega_{p}^{4} \tau_{p}^{2}}{4}-\frac{\omega_{p}^{8} \tau_{p}^{6}}{16}+O\left(\omega_{p}^{12} \tau_{p}^{10}\right)$ for $\tau_{p}^{4} \omega_{p}^{4} \ll 1$

\section{THE IMAGE-CHARGE FIELDS IN SPACE, TIME, AND FREQUENCY}

This section discusses the potentials due to an infinitely thin disk of uniform charge near the cathode surface. The analysis derives the time dependence of the image-charge electric field experienced by the disk as it accelerates from the cathode. Multiplying the Fourier transform of the image field times $g_{c}(\omega)$ gives the image-charge field as a function of the frequency. Transforming this product back to time gives the image-charge field as a function of the disk's time of travel from the cathode. This transformed image field which is traveling with the disk now includes the electronic response of the cathode.

\section{A. Potentials at the cathode surface and the Schottky effect}

Our model for the electron bunch is an infinitely thin disk of charge with a uniform charge density. The results given here can be integrated over a finite bunch length to give the fields for the ideal "beer can" bunch shape.

The derivation begins with the axial field of a solitary disk of charge as shown in Fig. 3. We obtain the imagecharge field by scaling the disk's charge by $q^{\prime} / q$ and placing it at the image charge's location behind the cathode. 


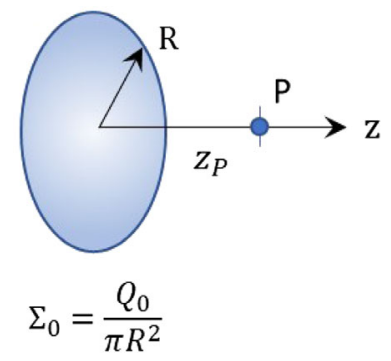

FIG. 3. Parameters of a thin disk of charge.

The field from this "image disk" is then computed at the real disk's $z$ position on the vacuum side. Assuming constant acceleration in an applied field gives the image-charge field at the disk vs the time of travel from the cathode.

The axial electric potential at point $P$ the distance $z_{P}$ from the surface of a disk with radius $R$ and uniform surface charge density $\Sigma_{0}$ is found to be [9]

$$
\begin{aligned}
V_{\text {disk }}\left(z_{P}\right) & =\frac{\Sigma_{0}}{2 \epsilon_{0}}\left(\sqrt{z_{P}^{2}+R^{2}}-\left|z_{P}\right|\right) \\
& =\frac{Q_{0}}{2 \pi \epsilon_{0} R^{2}}\left(\sqrt{z_{P}^{2}+R^{2}}-\left|z_{P}\right|\right) .
\end{aligned}
$$

Here, $R$ is the disk's radius and $z_{p}$ is the distance the axial point $P$ is from the disk center.

The image method places a copy of the real charge with opposite sign at the symmetrical position inside of the cathode to calculate the image-charge field it produces at the disk outside the cathode. Figure 4 shows the image method's configuration for a real-charge disk at $z=z_{s}$ and its image at $z=-z_{s}$. The disk and the image charge converge at the cathode surface as $z_{s} \rightarrow 0$. Shifting Eq. (13) to the image-charge location, using the surface loss function for $q^{\prime} / q$, and evaluating the potential at the disk center, $z_{P}=0$, gives the total potential energy at the center of the real-charge disk as

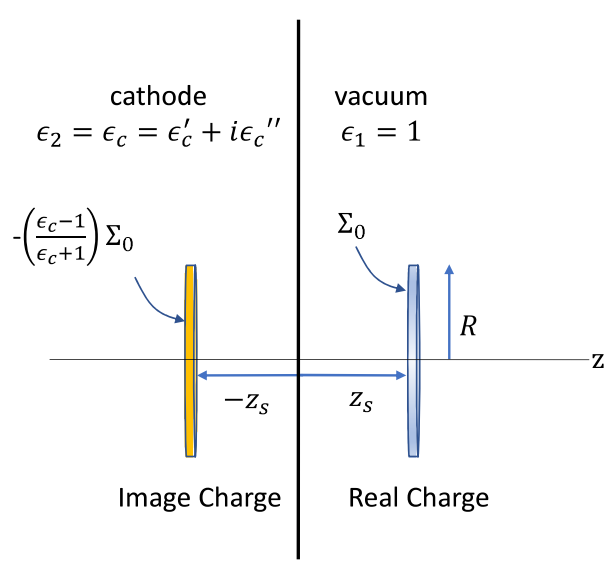

FIG. 4. The positions of a real disk of charge and its image with respect to the cathode surface.

$$
V_{\text {total }}\left(z_{s}\right)=-E_{a} z_{s}-\frac{Q_{0}}{\epsilon_{0} \pi R^{2}}\left(\frac{\epsilon_{c}-1}{\epsilon_{c}+1}\right)\left(\sqrt{z_{s}^{2}+\frac{R^{2}}{4}}-z_{s}\right)
$$

The first term on the right is the potential energy of the applied field. The second term is the image-charge potential energy at the center of a disk with radius $R$ and charge $Q_{0}$ located $z_{s}$ from the cathode surface.

Plots of the image-charge and the applied field potentials for disk radii of 50, 100, and $200 \mu \mathrm{m}$ are shown in Fig. 5. The disk charge is a 50-pC disk, and the applied field is $50 \mathrm{MV} / \mathrm{m}$. The figure shows that the potential well disappears for $R \geq 200 \mu \mathrm{m}$. And when the well exists, its peak is approximately $50 \mu \mathrm{m}$ from the cathode. The image potential is finite at $z_{s}=0$ and is equal to

$$
\begin{aligned}
V_{\text {image }}\left(z_{s}=0\right) & =-\frac{Q_{0}}{\epsilon_{0} 2 \pi R}\left(\frac{\epsilon_{c}-1}{\epsilon_{c}+1}\right) \\
& =\frac{Q_{0}}{\epsilon_{0} 2 \pi R} g_{c}(\omega) .
\end{aligned}
$$

The maxima of the potentials shown in Fig. 5 give the Schottky potentials for various radii disks near a metallic cathode. Setting the derivative of $V_{\text {total }}(z)$ equal to zero and solving for $z$ gives the potential barrier's maximum location, $z_{m}$, from the cathode surface. This distance is

$$
z_{m}=\frac{R}{2} \frac{1-\frac{E_{a}}{E_{\text {disk }}}}{\sqrt{1-\left(1-\frac{E_{a}}{E_{\text {disk }}}\right)^{2}}},
$$

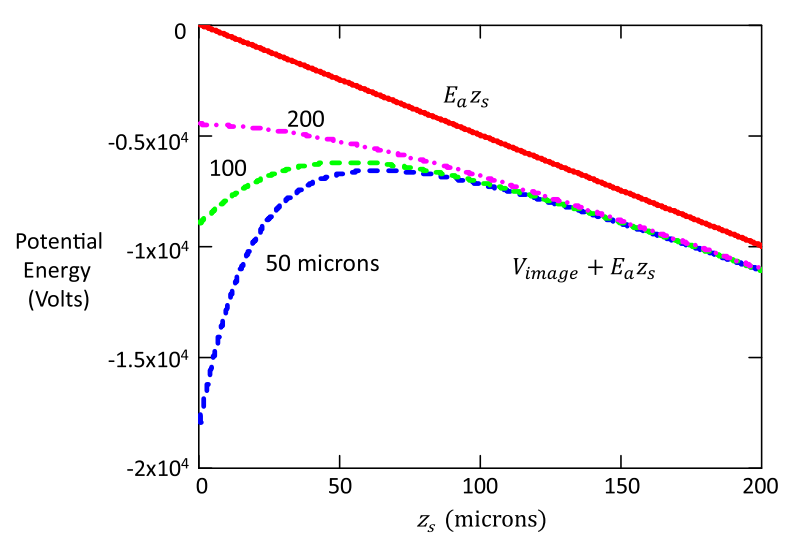

FIG. 5. The image-charge potential energy and the applied potential energy [as given by Eq. (14)] at the center of a 50-pC disk of charge as a function of its distance from a cathode with $q^{\prime}=-q$. The potential for an applied field of $50 \mathrm{MV} / \mathrm{m}$ is given by the solid red curve. The dashed curves are the potential energies for disks with 50-, 100-, and $200-\mu \mathrm{m}$ radii. Note that the maximum of the potential barrier is approximately $50 \mu \mathrm{m}$ from the cathode surface and moves toward the cathode with an increasing disk radius. 
where

$E_{\mathrm{disk}} \equiv \frac{Q_{0}}{\epsilon_{0} \pi R^{2}}\left(\frac{\epsilon_{c}-1}{\epsilon_{c}+1}\right)=\frac{Q_{0}}{\epsilon_{0} \pi R^{2}} g_{c}(\omega)=\frac{\Sigma_{0}}{\epsilon_{0}} g_{c}(\omega)$.

The potential well disappears when the applied field $E_{a}$ equals the image-charge field at the disk, $E_{\text {disk }}$. Evaluating $V_{\text {total }}$ at $z_{m}$ gives the Schottky potential for the disk of charge:

$V_{\text {Schottky }}\left(E_{a}, E_{\text {disk }}\right)=\phi_{\text {disk }}=\frac{R}{2} \frac{\frac{E_{a}}{e}\left(\frac{E_{a}}{E_{\text {disk }}}-2\right)}{\sqrt{1-\left(1-\frac{E_{a}}{E_{\text {disk }}}\right)^{2}}}$.

From these relations, we can establish the connection between the space charge limit and the cathode's surface loss function. In photocathode guns, a commonly used definition states that the SCL is reached when the field of the polarization charge density induced on the surface by the bunch (also known as the image field) equals the applied field. Equation (16) agrees with this definition, since $z_{m}=0$ and $\nabla V=0$ when $E_{\text {disk }}=E_{a}=E_{\mathrm{SCL}}$. Therefore, the SCL field depends upon the surface loss function:

$$
E_{\mathrm{SCL}}(\omega) \equiv \frac{Q_{0}}{\epsilon_{0} \pi R^{2}}\left(\frac{\epsilon_{c}-1}{\epsilon_{c}+1}\right)=\frac{Q_{0}}{\epsilon_{0} \pi R^{2}} g_{c}(\omega) .
$$

It is interesting to note that the image-charge potential of a single electron is similarly affected by the cathode's dielectric function. In this case, an electron's total potential energy including the cathode's surface loss function is

$$
\phi_{e}(z)=-e E_{a} z-\frac{e^{2}}{16 \pi \epsilon_{0}}\left(\frac{\epsilon_{c}-1}{\epsilon_{c}+1}\right) \frac{1}{z} .
$$

As described earlier, the Schottky potential is the total potential energy evaluated at its maximum, which is

$\phi_{\text {Schottky }}=-\sqrt{\frac{e E_{a}}{4 \pi \epsilon_{0}}\left(\frac{\epsilon_{c}-1}{\epsilon_{c}+1}\right)}=-\sqrt{\frac{e E_{a}}{4 \pi \epsilon_{0}} g_{c}(\omega)}$.

The relative permittivity of most cathode materials is large compared to 1 ; therefore, $g_{c} \approx 1$, and Eq. (21) reverts to the usual expression for the Schottky potential of a single electron.

\section{B. Time dependence of the image-charge fields}

We now calculate of the time-dependent image field at the center of the disk. The potential energy of the image charge at the center of a disk with uniformly distributed charge $Q_{0}$ a distance $z_{s}$ from a cathode surface is

$$
V_{\text {image }}\left(z_{s}\right)=-\frac{Q_{0}}{\epsilon_{0} \pi R^{2}}\left(\frac{\epsilon_{c}-1}{\epsilon_{c}+1}\right)\left(\sqrt{z_{s}^{2}+\frac{R^{2}}{4}}-z_{s}\right) .
$$

The divergence of the potential energy, $\vec{E}=-\vec{\nabla} V$, gives us the image charge's electric field acting on the center of the disk:

$E_{\text {image }}(t)=\frac{Q_{0}}{\epsilon_{0} \pi R^{2}}\left(\frac{\epsilon_{c}-1}{\epsilon_{c}+1}\right)\left(\frac{z_{s}(t)}{\sqrt{z_{s}^{2}(t)+\frac{R^{2}}{4}}}-1\right)$.

$z_{s}(t)$ is the disk's distance from the cathode at time $t$. If we assume the disk's motion is only due to its acceleration in the applied field, then

$$
z_{s}(t)=\frac{1}{\gamma^{\prime}}\left[\sqrt{\left(\gamma^{\prime} c t\right)^{2}+1}-1\right] .
$$

Here, $\gamma^{\prime} \equiv \frac{E_{a}}{m c^{2}}$ is the normalized applied field.

Inserting Eq. (24) into Eq. (23) and finding a closed expression for the Fourier transform seems impossible. Instead, a very good approximation for $E_{\text {image }}(t)$ is a heuristic Gaussian function. Setting the width and amplitude of a Gaussian equal to those for $E_{\text {image }}$ gives the following useful and accurate approximation:

$$
E_{\text {image }}^{\text {Gaussian }}(t)=-\frac{Q_{0}}{\epsilon_{0} \pi R^{2}}\left(\frac{\epsilon_{c}-1}{\epsilon_{c}+1}\right) e^{-\left(t^{2} / 2 \sigma_{t}^{2}\right)}, \quad t \geq 0 .
$$

The rms width of the Gaussian, $\sigma_{t}$, is the image-charge field duration, which is a function of the disk radius and the normalized applied field:

$$
\sigma_{t}^{2} \equiv \frac{\left(1+\gamma^{\prime} R / \sqrt{12}\right)^{2}-1}{(2 \ln 2) \gamma^{\prime 2} c^{2}} .
$$

Figure 6 shows that $E_{\text {image }}^{\text {Gausian }}$ closely matches $E_{\text {image, }}$, which justifies using it for $E_{\text {image }}$ in the remainder of this paper.

Figure 7 shows the image-charge field duration $\sigma_{t}$ as a function of the applied field for various disk radii. Clearly, the field's duration increases with larger disk radii and decreases at higher applied fields. Taking the limit of Eq. (26) as the applied field becomes infinite leads to a minimum value of the image field duration time for a given disk radius:

$$
\lim _{\gamma^{\prime} \rightarrow \infty} \sigma_{t}=\frac{R}{2 c \sqrt{\sqrt{3} \ln 2}} \cong 1.5 R[\mathrm{~mm}] \mathrm{ps} .
$$

Therefore, even with an infinitely large applied field, the electrons will experience the space-charge image field for approximately a picosecond unless the cathode radius size is very small. 


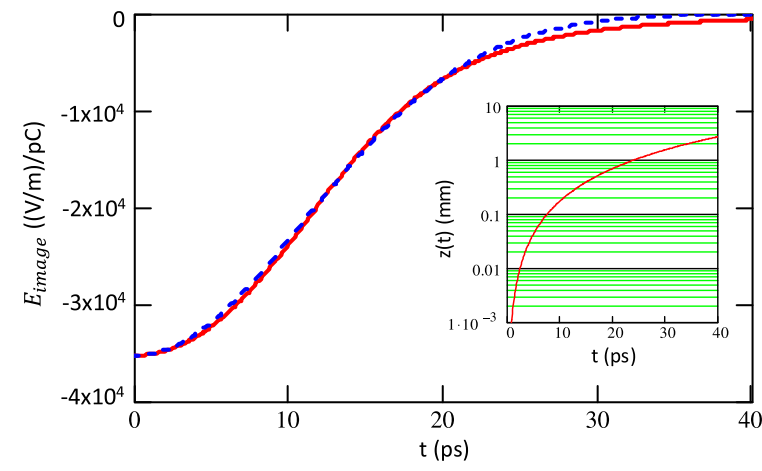

FIG. 6. Comparison of the image field given by Eqs. (23) and (24) (solid red curve) with the heuristic Gaussian function in Eq. (25) (dashed blue curve). The disk radius is $1 \mathrm{~mm}$, $E_{a}=20 \mathrm{MV} / \mathrm{m}$, and the cathode dielectric constant is 100 . The field is given per picocoulomb of negative disk charge. The inset shows the disk position from the cathode as a function of the time.

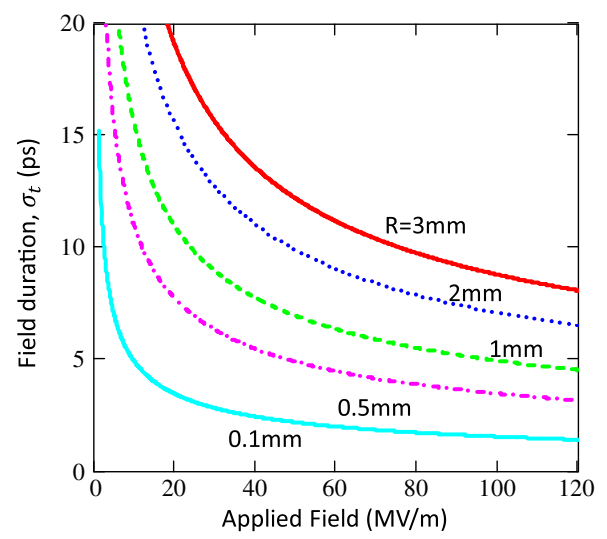

FIG. 7. The image field duration time as a function of the applied field for various disk radii.

\section{Frequency dependence of the image-charge fields}

Realizing that the image field is the product of frequency- and time-dependent functions, we make this separation explicit by writing the field as

$$
E_{\text {image }}^{\text {Gaussian }}(\omega, t)=\frac{Q_{0}}{\epsilon_{0} \pi R^{2}} g_{c}(\omega) f(t),
$$

where $g_{c}(\omega)$ is the cathode's surface loss function and $f(t)$ is defined as the Gaussian field shape function:

$$
f(t) \equiv e^{-\left(t^{2} / 2 \sigma_{t}^{2}\right)}
$$

The Fourier transform of $f(t)$ is another Gaussian given by

$$
\tilde{f}(\omega)=\sqrt{\frac{2}{\pi}} \sigma_{t} e^{-\left(\sigma_{t}^{2} \omega^{2} / 2\right)}
$$

with $1 / \sigma_{t}$ as the rms frequency spread of the transformed image-charge field. In this paper, the Fourier transform of a function is denoted by the function with a tilde symbol.

The Fourier transform of the image-charge field is thus the product of the surface loss function and the transformed field shape function:

$$
\tilde{E}_{\text {image }}^{\text {Gaussian }}(\omega)=\frac{Q_{0}}{\epsilon_{0} \pi R^{2}} g_{c}(\omega) \tilde{f}(\omega) .
$$

In this product, the surface loss function acts like a lowpass filter on $\tilde{f}(\omega)$, allowing only frequencies below the transition frequency to pass.

Since $\tilde{E}_{\text {image }}(\omega)$ can be represented by a Gaussian centered at $\omega=0$, it is convenient to define the highfrequency edge of $\tilde{E}_{\text {image }}(\omega)$ at its half-height as $\omega_{e}$. Thus, from Eq. (30),

$$
e^{-\left(\omega_{e}^{2} \sigma_{t}^{2} / 2\right)}=\frac{1}{2}
$$

Solving for the edge frequency $\omega_{e}$ gives

$\omega_{e}=\frac{\sqrt{2\left|\ln \frac{1}{2}\right|}}{\sigma_{t}}=2\left|\ln \frac{1}{2}\right| \sqrt{\frac{\gamma^{\prime 2} c^{2}}{\left(1+\gamma^{\prime} R / \sqrt{12}\right)^{2}-1}}$.

Thus, the edge frequency is a function of the disk radius and the applied field.

The temporal behavior and the magnitude of the imagecharge field depends upon the edge frequency relative to the transition frequency. There are three types of image fields depending upon $\omega_{t}$ with respect to $\omega_{e}$. They are as follows.

If $\omega_{t}>\omega_{e}$, then $q^{\prime} / q=-1$ with no imaginary part. This is true for all currently used cathodes.

If $\omega_{t}=\omega_{e}$, then $q^{\prime} / q$ has both real and imaginary parts. The imaginary part is at its maximum. The image-charge field strongly oscillates at the transition frequency, which could seed unwanted microbunching instabilities and reduce beam brightness or be used to prebunch the beam.

If $\omega_{t}<\omega_{e}$, then $q^{\prime} / q \sim 0$ for both real and imaginary parts. Without the image-charge field, there is no space charge limit to the emission.

\section{The image-charge electric field fourier transformed with the surface loss function}

Since $E_{\text {image }}(\omega, t)$ is an even function in time, the cosine representation for the Fourier transform is used [10]. Hence, the Fourier transform of the image field is given by

$$
\tilde{E}_{\text {image }}(\omega)=\frac{2}{\pi} \int_{0}^{\infty} E_{\text {image }}(\omega, t) \cos \omega t d t,
$$


and its transformation back to the time coordinate is denoted by the script of the function,

$$
\mathcal{E}_{\text {image }}(t) \equiv \int_{0}^{\infty} \tilde{E}_{\text {image }}(\omega) \cos \omega t d \omega
$$

The script $\mathcal{E}(t)$ is used to avoid confusion with the $E_{\text {image }}(t)$ which is already in Eq. (23). In addition, writing Eq. (35) with $E_{\text {image }}(t)$ could also cause confusion with $E_{\text {image }}(\omega, t)$, which is a function of two variables; see Eq. (28). $\mathcal{E}_{\text {image }}(t)$ includes the effects of the surface loss function, while $E_{\text {image }}(t)$ does not.

$\tilde{E}_{\text {image }}(\omega)$ is the Fourier spectrum of the image-charge field seen by the disk as it accelerates from the cathode. Assuming the Gaussian formulation for $E_{\text {image }}(t)$ is valid, the Fourier spectrum is easily found to be

$$
\tilde{E}_{\text {image }}^{\text {Gaussian }}(\omega)=-\sqrt{\frac{2}{\pi}} \frac{Q_{0}}{\epsilon_{0} \pi R^{2}}\left(\frac{\epsilon_{c}(\omega)-1}{\epsilon_{c}(\omega)+1}\right) \sigma_{t} e^{-\left(\omega^{2} \sigma_{t}^{2} / 2\right)} .
$$

Equation (36) gives the frequency spectrum of the image-charge field responding to the electrical impulse of the accelerating disk. Transforming $\tilde{E}_{\text {image }}^{\text {Gaussian }}(\omega)$ back to time gives the time-dependent image field modified by the cathode's response:

$$
\begin{aligned}
\mathcal{E}_{\text {image }}^{\text {Gaussian }}(t)= & -\sqrt{\frac{2}{\pi}} \frac{Q_{0}}{\epsilon_{0} \pi R^{2}} \sigma_{t} \int_{0}^{\infty}\left(\frac{\epsilon_{c}(\omega)-1}{\epsilon_{c}(\omega)+1}\right) \\
& \times e^{-\left(\omega^{2} \sigma_{t}^{2} / 2\right)} \cos \omega t d \omega .
\end{aligned}
$$

Using Drude's theory for the dielectric function, the integral can be written in terms of the plasma frequency and the relaxation time of the cathode surface:

$$
\begin{aligned}
\mathcal{E}_{\text {image }}^{\text {Gaussian }}(t)= & -\sqrt{\frac{2}{\pi}} \frac{Q_{0}}{\epsilon_{0} \pi R^{2}} \sigma_{t} \int_{0}^{\infty}\left(\frac{\omega_{p}^{2}}{\omega_{p}^{2}-2 \omega\left(\omega+\frac{i}{\tau_{p}}\right)}\right) \\
& \times e^{-\left(\omega^{2} \sigma_{t}^{2} / 2\right)} \cos \omega t d \omega .
\end{aligned}
$$

For a cathode with $\omega_{t} \gg \omega_{e}$, then $\epsilon_{c} \gg 1$; therefore, $\left(\frac{\epsilon_{c}-1}{\epsilon_{c}+1}\right) \rightarrow 1$ and $q^{\prime}=-q$. In this case, the Fourier transform of $\tilde{E}(\omega)$ returns the original image field such that $\mathcal{E}_{\text {image }}(t)=E_{\text {image }}(\omega=0, t)$, which is not a very interesting result. However, if one instead uses a cathode with a transition frequency below $\omega_{e}$, then some interesting effects can be discovered as will be described in Sec. IV.

\section{E. Transverse size of the polarization surface charge density}

In this subsection, the transverse size of the cathode necessary for the metasurface properties to dominate in electron emission is determined. The metasurface should be large enough to encompass all the polarization charge induced on the cathode's surface by the disk of charge. It is interesting to note that the polarization charge does not propagate or spread across the cathode. Instead, it is localized on the cathode surface beneath the emitted electrons.

Figure 8 shows the three views of the cathode-disk geometry in which an infinitesimal of charge, $d Q=$ $\Sigma_{0} r d r d \alpha^{\prime}$, located on the disk at coordinates $\left(r, \alpha^{\prime}\right)$ polarizes charge on the cathode surface at $(\rho, \alpha)$. The bracketed quantity in the integrand's denominator is the square of the three-dimensional distance between the points $(\rho, \alpha)$ on the cathode and $\left(r, \alpha^{\prime}\right)$ on the disk (Fig. 8, side view).

The polarization charge density on the cathode surface at radius $\rho$ induced by the infinitesimal charge $d Q$ at a distance $d$ from a surface and radius $\rho$ from the center of the disk is [4]

$$
d \sigma_{\mathrm{pol}}=-\frac{1}{2 \pi}\left(\frac{\epsilon_{c}-1}{\epsilon_{c}+1}\right)\left[\frac{d}{\left(\rho^{2}+d^{2}\right)^{3 / 2}}\right] d Q .
$$

The total polarization charge density at the point $(\rho, \alpha)$ on the cathode is found by integrating over the disk's charge distribution:

$$
\begin{aligned}
\sigma_{\mathrm{pol}}(\rho, \alpha)= & -\frac{d}{2 \pi}\left(\frac{\epsilon_{c}-1}{\epsilon_{c}+1}\right) \\
& \times \int_{0}^{2 \pi} \int_{0}^{R} \frac{\Sigma_{0} r d r d \alpha^{\prime}}{\left[\rho^{2}+d^{2}+r^{2}-2 \rho r \cos \left(\alpha-\alpha^{\prime}\right)\right]^{3 / 2}} .
\end{aligned}
$$

Because of the disk's axial symmetry, there is no loss of generality setting $\alpha=0$ and determining the charge density profile along the $x$ axis. Doing this and performing the radial integral gives

$$
\begin{aligned}
& \sigma_{\mathrm{pol}}(\rho, \alpha=0) \\
& =-\frac{d}{2 \pi}\left(\frac{\epsilon_{c}-1}{\epsilon_{c}+1}\right) \Sigma_{0} \int_{0}^{2 \pi}\left\{-\frac{s^{2}-\rho R \cos \alpha^{\prime}}{\left(R^{2}+s^{2}-2 \rho R \cos \alpha^{\prime}\right)^{1 / 2}}+s\right\} \\
& \quad \times \frac{d \alpha^{\prime}}{s^{2}-\rho^{2} \cos ^{2} \alpha^{\prime}},
\end{aligned}
$$

where $s^{2}=\rho^{2}+d^{2}$. This expression is numerically integrated to obtain the polarization charge density.

Figure 9 shows the polarization charge density along the $x$ axis for a 100-pC, 1-mm radius disk positioned 0.03, 0.3, and $3 \mathrm{~mm}$ from the cathode. The figure indicates that the 


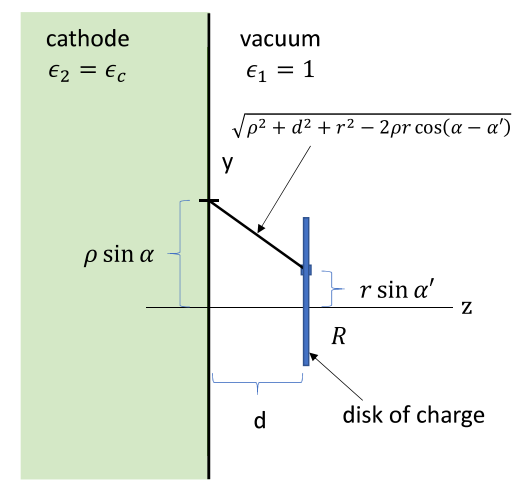

side view
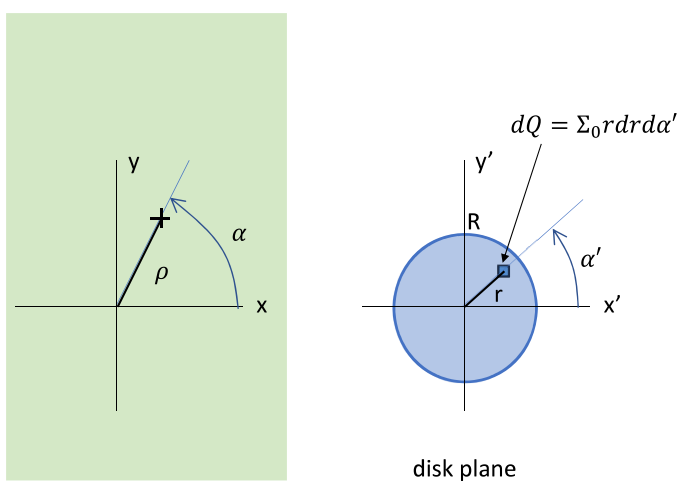

disk plane

cathode-vacuum plane

FIG. 8. The geometry for calculating the polarization charge density on the cathode surface. The side view shows the distance between the point $(\rho, \alpha)$ on the cathode surface and the point $\left(r, \alpha^{\prime}\right)$ on the disk. The disk has a uniform surface charge density of $\Sigma_{0}$. The polarization charge density at the point on the cathode is given by integrating the disk's surface charge density over $r$ and $\alpha^{\prime}$. The $x$ axis is into the page in the side view, and the $z$ axis is out of the page in the cathode-vacuum and disk plane views.

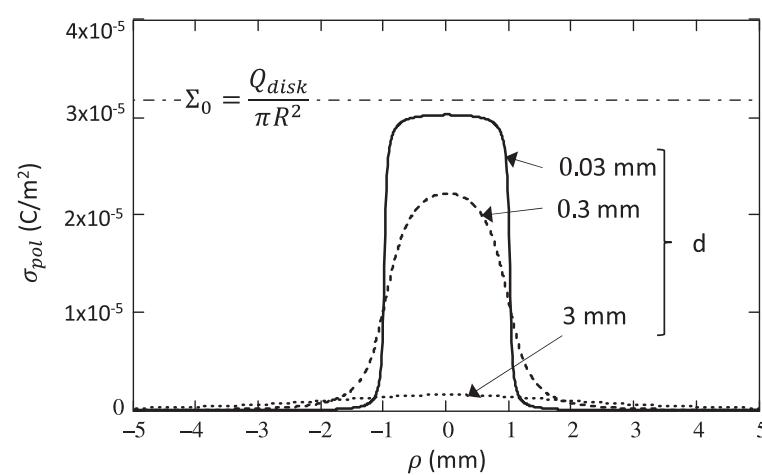

FIG. 9. The polarization charge density on the cathode surface for a $R=1 \mathrm{~mm}$ radius disk with $Q_{\text {disk }}=100 \mathrm{pC}$. The calculations assume $q^{\prime}=-q$. Lineout profiles of the surface charge density are shown for the disk at $d=0.03,0.3$, and $3 \mathrm{~mm}$ from the cathode. The surface charge density of the disk, $\Sigma_{0}$, is shown with a horizontal dash-dotted line.

transverse extent of the polarization charge is about the same as the disk size, which in this case is $2 \mathrm{~mm}$ in diameter. From this transverse distribution, we conclude that the metasurface should be at least twice the emission area to include tails of the distribution.

Because the thickness of the polarized layer is only a few angstroms [11], one would assume that the metacathode can be made very thin. However, the actual thickness will depend upon the type of metasurface and its surface loss function. This is discussed with more detail in Sec. IV B.

\section{CATHODE DESIGN WITH METASURFACES}

A metasurface is a surface composed of a pattern of structures, each of which is smaller than the wavelength of the radiation of interest. For photon wavelengths longer than the structure spacing and size, these artificial atoms interact with radiation like atoms in an atomic lattice. Such an artificial "atomic" structure can have significantly different electronic properties than those of natural bulk materials. Varying the type of structures and their patterns produces a wide range of electrical and magnetic properties, including many which are otherwise unobtainable in nature. In the case of cathode design, an important electronic property is the dielectric function which affects the electron's initial dynamics via the surface loss function. The metasurface's dielectric function is determined by its structure, and, as will be shown, the metasurface can be structured to mitigate the cathode's SCL.

\section{A. The surface loss function and general behavior of a wire-array metasurface}

Studies of the electrical properties of "artificial dielectrics" began in the 1940s. An early description of wire arrays in terms of a plasma was given by Rotman, who simulated microwave antennas consisting of 1D, 2D, and 3D wire arrays [12]. More recently, wire arrays which mimic materials with a very low plasma frequency are discussed by Pendry et al. [13]. In this work, Pendry describes a 3D cubic lattice of wires and emphasizes the importance of the wire radius being much smaller than the wire spacing.

Here, we study a 1D metamaterial consisting of a rectangular pattern of thin metal wires aligned parallel to the $z$ axis. The direction of the wires determines the electric field polarization coupling to the array's dielectric function. If the wires are thin compared to the wire spacing, the array is nearly transparent to the $x$ and $y$ components of the applied field. Adding thin wires aligned parallel to the $x$ and $y$ axes would make a 3D metamaterial which would couple to all three components of the applied electric field. The elements of the $3 \times 3$ diagonal dielectric function tensor would be determined by the wire spacings, radii, and conductivities of the wires parallel to each of the three axes' directions. 


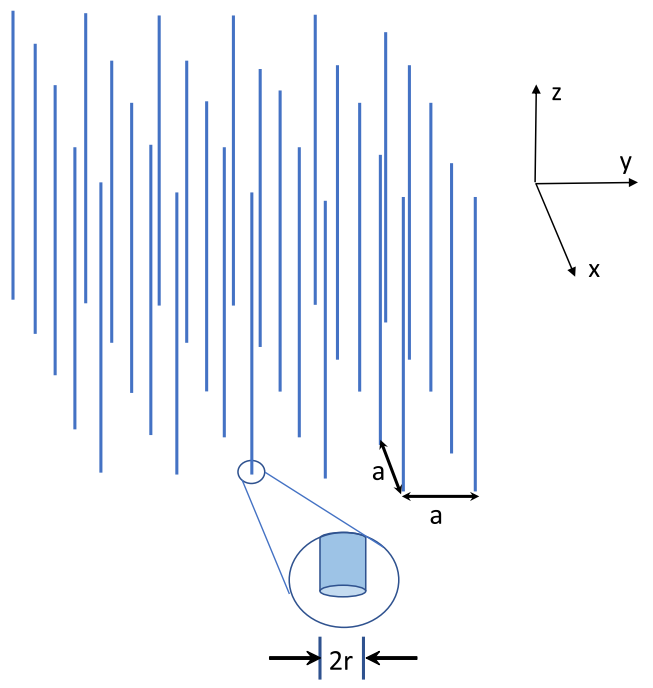

FIG. 10. A 1D wire-array metamaterial consisting of a rectangular array of parallel wires with spacing $a$ and wire radius $r$. These dimensions combined with the wire conductivity $\sigma_{\text {wire }}$ specify the plasma frequency and the relaxation time of the metamaterial. Since the wires are aligned along the $z$ axis, it is only the $z$ component of the electric field which can electrically polarize the wires. The $x$ - and $y$-field components are unaffected by the array.

The 1D metamaterial shown in Fig. 10 has a plasma frequency with $z$ polarization given by [14]

$$
\omega_{\mathrm{wire}}^{2}=\frac{2 \pi c^{2}}{a^{2} \ln \frac{a}{r}}
$$

and relaxation time

$$
\tau_{\text {wire }}=\frac{r^{2} \sigma_{\text {wire }}}{2 \epsilon_{0} c^{2}} \ln \frac{a}{r}
$$

Here, $a$ is the center-to-center distance between the wires, $r$ is the wire radius, and $c$ is the vacuum speed of light. The conductivity of each wire is $\sigma_{\text {wire }}$, and the host material between the wires is assumed to be a vacuum. The objective is to find values for the three metasurface parameters $(a, r$, and $\sigma_{\text {wire }}$ ) which reduce the transition frequency by 6 orders of magnitude from $10^{16}$ to $10^{10} \mathrm{rad} / \mathrm{s}$. To do this, we fix the wire radius and the wire conductivity and vary the wire spacing to lower $\omega_{t}$.

Table II gives the characteristics of metasurfaces with wire spacings corresponding to the three frequency regions described earlier, and Fig. 11 shows their image-charge fields and $-g_{c}(\omega)$. For all three spacings, the wire radius is $1 \mu \mathrm{m}$, and the wire conductivity is equal to that of copper. The applied field is $50 \mathrm{MV} / \mathrm{m}$, which combined with a $R=1 \mathrm{~mm}$ disk radius gives $\sigma_{t}=6.9 \mathrm{ps}-\mathrm{rms}$. The edge frequency is $\omega_{e}=1.7 \times 10^{11} \mathrm{rad} / \mathrm{s}$. The wire spacings $a=0.5,1.3$, and $10 \mathrm{~mm}$ correspond to $\omega_{t}>\omega_{e}$,
TABLE II. Parameters of rectangular arrays of parallel wires for three wire spacings. The wire conductivity is $\sigma_{\text {wire }}=$ $6.3 \times 10^{7} \mathrm{~S} / \mathrm{m}$, and the wire radius is $r=1 \mu \mathrm{m}$ for all three spacings. The image field duration or transit time is $\sigma_{t}=6.9 \mathrm{ps}$. The applied field is $50 \mathrm{MV} / \mathrm{m}$.

\begin{tabular}{lccc}
\hline \hline Frequency range & $\omega_{t}>\omega_{e}$ & $\omega_{t} \cong \omega_{e}$ & $\omega_{t}<\omega_{e}$ \\
\hline $\begin{array}{l}\text { Wire spacing } a(\mathrm{~mm}) \\
\text { Array plasma freq. }\end{array}$ & 0.5 & 1.3 & 10 \\
$\quad \omega_{\text {wire }}(\mathrm{rad} / \mathrm{s})$ & $6.0 \times 10^{11}$ & $2.2 \times 10^{11}$ & $2.5 \times 10^{10}$ \\
$\begin{array}{l}\text { Relaxation time } \\
\quad \tau_{\text {wire }}(\mathrm{s})\end{array}$ & $2.5 \times 10^{-10}$ & $2.8 \times 10^{-10}$ & $3.6 \times 10^{-10}$ \\
$\begin{array}{l}\text { Transition frequency } \\
\quad \omega_{t}(\mathrm{rad} / \mathrm{s})\end{array}$ & $4.3 \times 10^{11}$ & $1.5 \times 10^{11}$ & $1.7 \times 10^{10}$ \\
$\begin{array}{l}\text { Edge frequency } \\
\omega_{e}(\mathrm{rad} / \mathrm{s})\end{array}$ & $1.7 \times 10^{11}$ & $1.7 \times 10^{11}$ & $1.7 \times 10^{11}$ \\
\hline \hline
\end{tabular}

$\omega_{t} \cong \omega_{e}$, and $\omega_{t}<\omega_{e}$, respectively. The image-charge fields in Fig. 11 follow the behavior described in Sec. III C for these three frequency ranges.

\section{B. Practical metacathode design}

Although instructive, the wire arrays with millimeter spacing described in the last subsection are not practical cathode designs for high-brightness electron guns. A realistic metacathode would have a wire-to-wire spacing of microns in order to have thousands of wires over a millimeter size area. This thousandfold reduction (millimeters to microns) in spacing is possible by simultaneously shrinking the wire radius and reducing the wire conductivity. In this miniaturized design, the metacathode becomes an array of hundreds of thousands of low conductivity nanowires (NWs).

There are two surface loss regimes which decide how the thousandfold reduction in the wire spacing can be achieved, the low-loss and high-loss regimes. [See the discussion in Sec. II for Eqs. (10)-(12).] In the low-loss or long relaxation time regime, the real part of the surface loss function is larger than the imaginary part, and in the high-loss or short relaxation time regime, the imaginary part dominates.

The low-loss or long relaxation time regime occurs when $\tau_{p} \omega_{p} \gg 1$ and has a transition frequency equal to $\omega_{p} / \sqrt{2}$ or

$$
\omega_{t}=\frac{c}{a} \sqrt{\frac{\pi}{\ln \frac{a}{r}}} \text { for } \tau_{p} \omega_{p} \gg 1 .
$$

Therefore, for low-loss metasurfaces, low transition frequencies can be obtained only by making the wire spacing large. This point was illustrated in Fig. 11, where a spacing of $10 \mathrm{~mm}$ was needed to lower the transition frequency enough to suppress the image field.

On the other hand, for a high-loss metasurface, the relaxation time is short compared to a plasma period, e.g., 

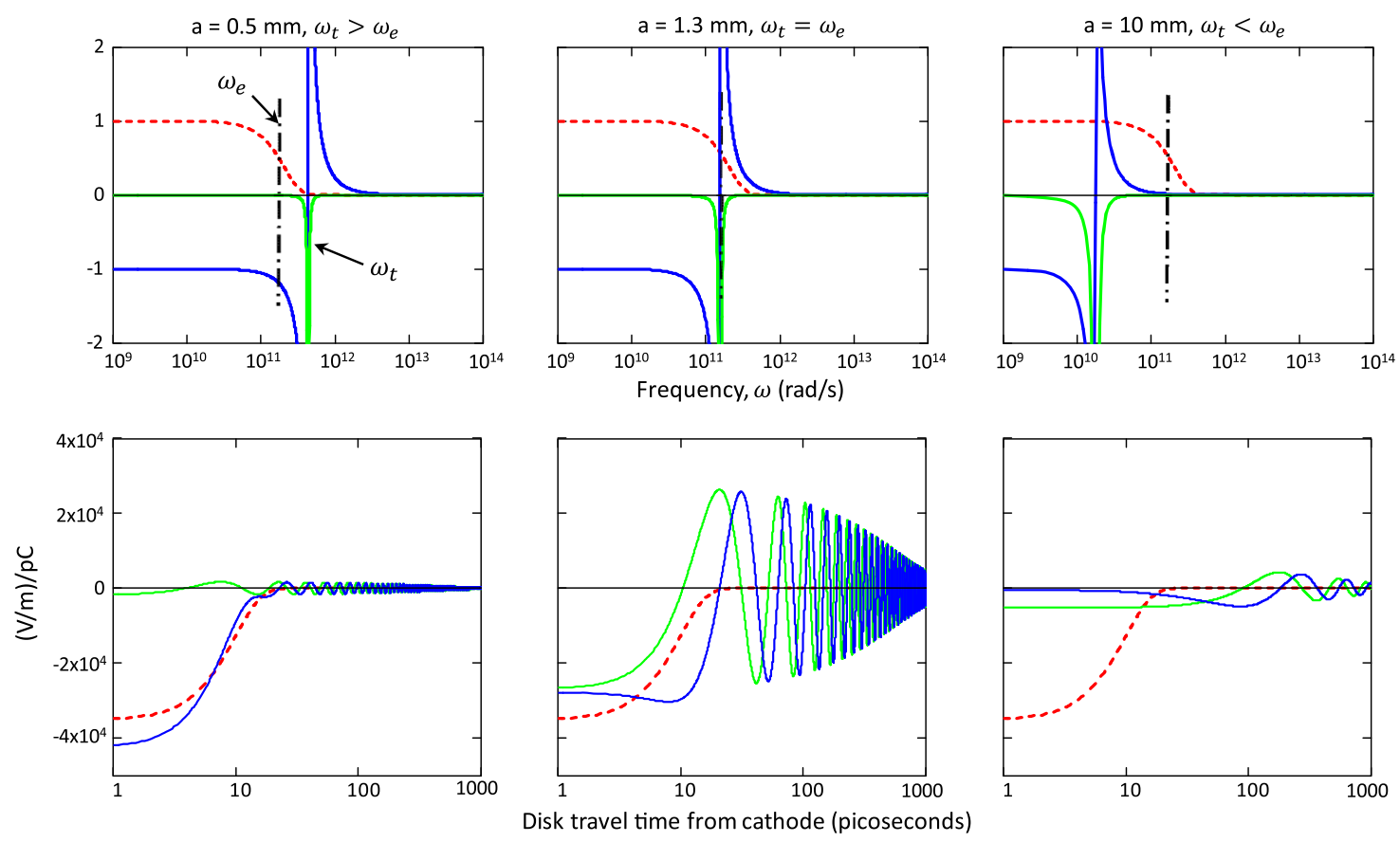

FIG. 11. The frequency spectra and the image-charge fields vs the time for three wire array metamaterials with spacings of $a=0.5$, 1.3 , and $10 \mathrm{~mm}$ corresponding to $\omega_{t}>\omega_{e}, \omega_{t} \cong \omega_{e}$, and $\omega_{t}<\omega_{e}$, respectively. The other array characteristics are given in Table II. The image field and its frequency spectrum for $q^{\prime}=-q$ are plotted with red dashed lines for reference and comparison. The applied field is not shown. Upper plots: The image-charge field frequency spectrum normalized at $\omega=0$ and the real and imaginary parts of the surface loss function. The vertical dash-dotted line is the edge frequency at $\omega_{e}=1.7 \times 10^{11} \mathrm{rad} / \mathrm{s}$. Red dashed curve: $\frac{1}{\sigma_{t}} \sqrt{\frac{\pi}{2}} \tilde{f}(\omega)$. Blue solid curve:- $\operatorname{Re} g_{c}(\omega)$. Green solid curve: $-\operatorname{Im} g_{c}(\omega)$. Lower plots: The image-charge field per pC at the disk center. Blue solid curve: $\operatorname{Re} \mathcal{E}_{\text {image }}^{\text {Gausian }}(t)$; green solid curve: $\operatorname{Im} \mathcal{E}_{\text {image }}^{\text {Gausian }}(t)$; red dashed curve: $E_{\text {image }}(t)$ with $q^{\prime}=-q$.

$\tau_{p} \omega_{p} \ll 1$, and the transition frequency is instead given by Eq. (12). In this case, the transition frequency scales according to

$$
\omega_{t} \propto \frac{r \sqrt{\sigma_{\text {wire }}}}{a} \text { for } \tau_{p} \omega_{p} \ll 1 .
$$

In this case, it is possible to lower the transition frequency by reducing $r \sqrt{\sigma_{\text {wire }}}$. Therefore, by greatly shrinking both the wire radius and conductivity, one can achieve low transition frequencies with micron scale wire spacings.

\section{Review of the nanowire technology and its application to metacathode design}

The previous subsection has shown that wire radii of tens of nanometers and wire spacings of a few microns are needed for a realistic metacathode. In this subsection, it is shown that such nanowire dimensions are well within the fabrication capability of contemporary silicon lithography. This technology has demonstrated the fabrication of dense rectangular arrays of freestanding, microns-tall silicon pillars. The process of template-based metalassisted chemical etching [15] has been used to produce vertically aligned wires as small as $5 \mathrm{~nm}$ in diameter [16]. Control of the precise positioning, radius, and length of the nanowires has been demonstrated using electron beam lithography and inductively coupled-plasma reactive ion etching $[17,18]$. In fact, silicon nanowires (SiNWs) with a height to diameter aspect greater than 200 have been achieved with a high density over a $1 \times 1 \mathrm{~mm}$ area [16]. Therefore, $10-\mathrm{nm}$ diameter nanowires could be as tall as $2 \mu \mathrm{m}$.

The electrical conductivity of the SiNWs can be controlled by the type and amount of doping used. The resistivity of silicon at room temperature is $2300 \Omega \mathrm{m}$, which is much higher than the desired resistance of $0.028 \Omega \mathrm{m}$ (see Table III). However, the semiconductor industry has shown that the resistivity of silicon can be precisely controlled in the $0.01-0.1 \Omega \mathrm{m}$ range with $\mathrm{p}$ doping [16].

In other work on a vertical field effect transistor (FET) [19], researchers created a SiNW FET which has NW dimensions and resistivity compatible with our metacathode design. The conducting channel of the SiNW FET is a $60-\mathrm{nm}$ diameter, $1.7-\mu$ m-long $\mathrm{SiNW}$ coated with an insulating layer of 25-nm-thick $\mathrm{SiO}_{2}$. The $\mathrm{SiNW}$ resistivity is $0.028 \Omega \mathrm{m}$ (hole concentration of $4 \times 10^{15} \mathrm{~cm}^{-3}$ ), which is also acceptable for the resistivity of the metacathode NWs. The gate electrode is a 100-nm-thick layer of aluminum covering the lower micron of the wire. In these 
TABLE III. The nanowire specifications and metasurface and beam parameters for the metacathode. The NW specifications are based upon demonstrated SiNW properties [19].

\begin{tabular}{|c|c|c|}
\hline \multicolumn{3}{|c|}{ Nanowire specifications } \\
\hline Tip coating & \multicolumn{2}{|c|}{$\begin{array}{c}\text { 5-nm of DLC or monolayer } \\
\text { of diamondoid }\end{array}$} \\
\hline Tip shape & Ellipsoid & 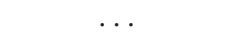 \\
\hline Minor axis radius $b$ & 30 & Nanometers \\
\hline Spacing $a$ & 2.0 & Microns \\
\hline Length $h$ & 1.35 & Microns \\
\hline Field enhancement factor & 400 & $\cdots$ \\
\hline Resistivity $\rho_{\text {wire }}$ & 0.028 & $\Omega \mathrm{m}$ \\
\hline Conductivity $\sigma_{\text {wire }}$ & 36 & $\Omega^{-1} \mathrm{~m}^{-1}$ \\
\hline Resistance $R_{\text {wire }}$ & 13 & $\mathrm{M} \Omega$ \\
\hline \multicolumn{3}{|c|}{ Metasurface parameters } \\
\hline Active cathode area & $1 \mathrm{~mm} \times 1 \mathrm{mn}$ & \\
\hline Wire density & $2.5 \times 10^{5}$ & nanowires $/ \mathrm{mm}^{2}$ \\
\hline Array plasma frequency $\omega_{\text {wire }}$ & $1.8 \times 10^{14}$ & $\mathrm{rad} / \mathrm{s}$ \\
\hline Relaxation time $\tau_{\text {wire }}$ & $8.3 \times 10^{-5}$ & Femtoseconds \\
\hline Array transition frequency $\omega_{t}$ & $1.4 \times 10^{9}$ & $\mathrm{rad} / \mathrm{s}$ \\
\hline \multicolumn{3}{|c|}{ Beam parameters } \\
\hline Cathode radius $R$ & 1 & $\mathrm{~mm}$ \\
\hline Cathode applied field $E_{a}$ & 5 & $\mathrm{MV} / \mathrm{m}$ \\
\hline Normalized applied field $\gamma^{\prime}$ & 9.8 & $\ldots$ \\
\hline Image-charge field duration $\sigma_{t}$ & 22 & Ps \\
\hline $\begin{array}{l}\text { Image-charge field edge } \\
\text { frequency } \omega_{e}\end{array}$ & $5.4 \times 10^{10}$ & $\mathrm{rad} / \mathrm{s}$ \\
\hline
\end{tabular}

experiments, a metal patch on the SiNW's top was used as the drain electrode for the current measurements.

Measurements of the SiNW vertical FET showed a current of $18 \mu \mathrm{A}$ through the SiNW for a gate bias of $-4 \mathrm{~V}$ [19]. This current is due to the impact ionization field effect [20], which is controlled by the Al gate. If one considers each SiNW in a $2-\mu \mathrm{m}$ space array emitting this current, then the total current density would be $28 \mu \mathrm{A} /$ wire $\times 2.5 \times 10^{5}$ wires $/ \mathrm{mm}^{2}=7 \mathrm{~A} / \mathrm{mm}^{2}$.

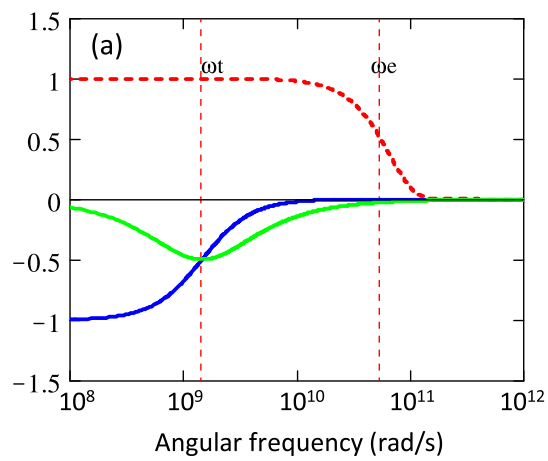

This current is much lower than the limiting current due to the resistance of the 60-nm SiNW. The resistance-limited current $I_{\text {wire }}$ is the voltage across the wire divided by the wire's resistance. For a large tip field enhancement, the voltage across a wire which is $h$ long and with $\beta_{\text {tip }}$ for the tip's field enhancement factor is $\sim \beta_{\text {tip }} E_{a} h$. A SiNW with the dimensions and conductivity given in Table III has a resistance of $13 \mathrm{M} \Omega$ and $\beta_{\text {tip }}=400$ (see Sec. V). Therefore, the SiNW resistance-limited current with an applied field of $5 \mathrm{MV} / \mathrm{m}$ is

$$
I_{\text {wire }}=\frac{V_{\text {tip }}}{R_{\text {wire }}}=\frac{\beta_{\text {tip }} E_{a} h}{R_{\text {wire }}}=208 \mu \mathrm{A} / \mathrm{SiNW} .
$$

Since there are $2.5 \times 10^{5} \mathrm{SiNWs} / \mathrm{mm}^{2}$, the resistancelimited current density is $52 \mathrm{~A} / \mathrm{mm}^{2}$. The resistance-limited photoelectric surface charge density of a 10-ps-long laser pulse would be $520 \mathrm{pC} / \mathrm{mm}^{2}$.

This charge density is far beyond the space charge limit of a cathode at $5 \mathrm{MV} / \mathrm{m}$. As discussed earlier, the SCL occurs when $\mathcal{E}_{\text {image }}=E_{a}$. Then in terms of the image field per charge, $\mathcal{E}_{\text {image }} / q$, the image field limited charge is

$$
Q_{\mathrm{SCL}}=\frac{E_{a}}{\mathcal{E}_{\text {image }} / q}
$$

Figure 12(b) shows that for a $q^{\prime}=-q$ cathode at $5 \mathrm{MeV} / \mathrm{m}$ (dashed red curve) the SCL charge density is $143 \mathrm{pC} / \mathrm{mm}^{2}$, since $\mathcal{E}_{\text {image }} / q=-3.5 \times 10^{4}(\mathrm{~V} / \mathrm{m}) / \mathrm{pC}$, whereas for the metacathode $\mathcal{E}_{\text {image }} / q$ is $-2.5 \times 10^{3}(\mathrm{~V} / \mathrm{m}) / \mathrm{pC}$. Therefore, the metacathode's SCL charge is 14 times larger or $2 \mathrm{nC} / \mathrm{mm}^{2}$. And, as just shown, this is well above the resistance-limited charge density of $520 \mathrm{pC} / \mathrm{mm}^{2}$ for the SiNW array. Therefore, the metacathode produces negligible image fields, and from the beam's perspective the cathode disappears.

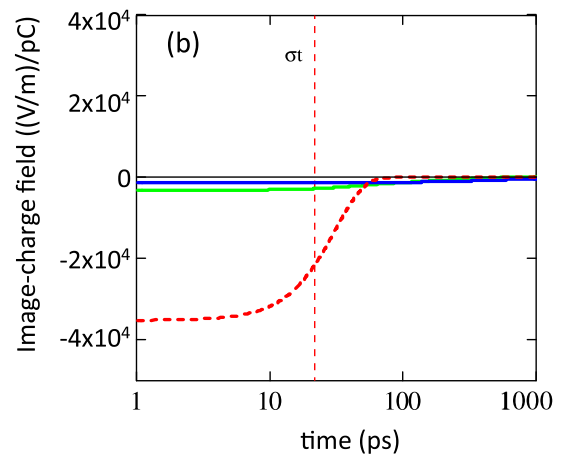

FIG. 12. (a) The Fourier spectrum of the image field shape function $\tilde{f}(\omega) / \tilde{f}(0)$ (red dashed curve) as a function of the angular frequency. The edge frequency is shown at $\omega_{e}$. The real (blue solid curve) and imaginary (green solid curve) parts of the cathode surface loss function $-g_{c}(\omega)$. The transition frequency is indicated at $\omega_{t}$. (b) The real (blue solid curve) and imaginary (green solid curve) parts of the image-charge field of the metacathode. The image-charge field per $\mathrm{pC}$ for the standard cathode (red dashed curve) is shown for reference. All curves are calculated using the design parameters given in Table III. 
In this design, it is assumed that all the nanowire tips are electron emitters. However, because of their nanometer size, the wires will exhibit strong quantum effects, which will confine the electrons and limit how many will be emitted. The quantized states in the quantum well (QW) on the surface are filled by electrons tunneling through from the silicon substrate. However, given the dimensions of most QWs, the energy of the lowest-lying quantum state is high in the conduction band, where there are very few electrons available to fill the QW and subsequently be emitted. Therefore, the typical QW can have limited charge emission. Fortunately, these detrimental quantum effects can be turned to our advantage by utilizing phenomena like quantum resonance tunneling to enhance the emission.

Because of field penetration into the QW, an external field (with the proper polarity) can bend the QW's states downward in energy to the bottom of the SiNW's conduction band, where there are more electrons. Those conduction electrons at the energy of the quantum state will resonantly tunnel though the SiNW-QW barrier and fill the quantum state. This quantum state then quickly decays by electron emission, because the applied field also lowers the second QW-vacuum barrier.

To summarize SiNW photoemission at a high applied field, electrons are photoexcited from the SiNW valence band to the conduction band, and these electrons then resonantly tunnel through the SiNW-QW barrier to the bent quantum state and onto the vacuum through the QWvacuum barrier. In this manner, the quantum state acts like a "wire" resonantly conducting electrons from the SiNW's conduction band to the vacuum.

Further details of resonant tunneling are given by Litovchenko et al. [21], whose research shows that a quantum well forms within a 5-nm-thick diamondlike carbon (DLC) layer with a 1.5-eV-high barrier between the silicon nanowire and the DLC. Both theoretical and experimental field emission studies exhibit a prominent peak in the field emission current when the applied field is approximately $2 \times 10^{9} \mathrm{~V} / \mathrm{m}$ due to enhanced transmission through the quantum state as just described. Therefore, to take advantage of this effect, the SiNW tip should be coated with a 5-nm layer of DLC and a field enhancement factor and applied field necessary to produce a $2 \mathrm{GV} / \mathrm{m}$ tip field. This field is easily achieved even at a low applied field because of the tip's large field enhancement factor. The engineering of the SiNW and its tip are discussed in the next two sections.

\section{METACATHODE DESIGN PARAMETERS AND CHARACTERISTICS}

The metacathode design is based upon the capabilities of current nanometer-scale lithography technology as reported in the literature. To add confidence to the design concept, the metacathode specifications given in Table III are the same as those of the SiNW FET described in Sec. IV.
The published results of the SiNW FET show that the NW dimensions and properties needed for the metacathode design are realistic and can be fabricated using proven lithographic techniques. Therefore, given the discussion of the last section, the metacathode will be made of 30-nm radius silicon nanowires with a resistivity of $0.028 \Omega \mathrm{m}$ and wire separation of $2 \mu \mathrm{m}$.

This leaves the SiNW's length left to determine. While the theory only loosely specifies the wire's length by simply requiring it should be longer than $c \tau_{\text {wire }}$, a more specific requirement can be established by considering the $\beta_{\text {tip }}$ needed to achieve a tip field of $2 \mathrm{GV} / \mathrm{m}$. And, although the radius is constrained by the surface loss function, one can freely adjust the wire height to obtain any desired field enhancement factor.

In addition to the wire's height-to-radius ratio, the tip's field enhancement also depends upon the shape of the tip. The field enhancement of a tip with a spherical shape is much lower than for an ellipsoid shape given the same wire height-to-radius ratio [22]. For example, a spherical tip wire with $h / r=100$ has a field enhancement factor of $\sim 100$, while for an ellipsoid tip with $h / r=100$ the field enhancement is $\sim 5000$. This sensitivity to tip shape expands the range of tip fields a given applied field can produce. In other words, high cathode field guns require less tip field enhancement and, therefore, the tip should have a spherical shape, while in low cathode field guns the tip shape should be an ellipsoid to increase the field enhancement. These details of the tip's shape become important if resonant tunneling is used to enhance the emission.

According to the work of Litovchenko et al. [21], an applied field of $2 \mathrm{GV} / \mathrm{m}$ is necessary to bend the quantum state energies of a QW inside a 5-nm-thick layer of DLC down to the bottom of the conduction band. The SiNW tip field is given by

$$
E_{\text {tip }}=\beta_{\text {tip }} E_{a} .
$$

Therefore, with $5 \mathrm{MV} / \mathrm{m}$ for the design applied field, the tip field enhancement factor necessary to achieve $E_{\text {tip }}=2 \mathrm{GV} / \mathrm{m}$ is

$$
\beta_{\text {tip }}=\frac{E_{\text {tip }}}{E_{a}}=400 .
$$

As explained earlier, either a spherical or ellipsoidal tip shape can be used to obtain the same field enhancement factor; however, their aspect ratios will be very different. Referring to the literature [22], one finds the following wire height-to-radius ratios for the two shapes which give the same field enhancement factor:

$$
\frac{h}{r}=500 \text { for a spherical tip }
$$

and 


$$
\frac{h}{b}=45 \text { for an ellipsoid tip, }
$$

where $h$ is the wire height, $r$ is the radius of the spherical tip, and $b$ is the length of the semiminor axis of the ellipsoid. The major axis of the ellipsoid is aligned along the wire's center line.

Equation (48) indicates that a spherical tip is not technically feasible, because an applied field of $5 \mathrm{MV}$ requires the extreme aspect ratio of 500 to achieve $\beta_{\text {tip }}=400$. However, the aspect needed for the ellipsoid tip [Eq. (49)] is 10 times less and has been demonstrated $[15,16]$. Therefore, an ellipsoid is selected for the tip shape. Hence, with $b=30 \mathrm{~nm}$, the SiNW height is $1.35 \mu \mathrm{m}$.

Table III summarizes the metacathode design specifications and parameters which are grouped into the three categories of nanowire, metasurface, and beam.

The wire and array values in Table III are used to compute curves shown in Fig. 12. The upper portion (positive abscissa values) in Fig. 12(a) shows the Fourier transform of the Gaussian field shape function [Eq. (29)] of the electrons as they accelerate from the cathode. The spectrum is constant from zero frequency out to the edge frequency $\omega_{e}=5.4 \times 10^{10} \mathrm{rad} / \mathrm{s}$.

The lower portion (negative abscissa values) in Fig. 12(a) plots the real and imaginary parts of $q^{\prime} / q$ [also known as $\left.-g_{c}(\omega)\right]$ for the metacathode. The real and imaginary parts of the surface loss function are highly broadened at the transition frequency, since this is a high-loss metasurface with $\tau_{p} \omega_{p} \ll 1$. The transition frequency is $\omega_{t}=1.4 \times 10^{9} \mathrm{rad} / \mathrm{s}$; hence, $\omega_{t} \ll \omega_{e}$, and the image field is reduced a factor of 14 .

Figure 12(b) compares the image fields of a normal cathode $\left(q^{\prime}=-q\right)$ with the metacathode design given in Table III. The normal cathode image field is $\sim 3.5 \times 10^{4}(\mathrm{~V} / \mathrm{m}) / \mathrm{pC}$, while the metacathode's is $2.5 \times 10^{3}$. This is a 14-fold reduction in the image field.

And, finally, the tip of each SiNW should be coated with a work function reducing material in order to improve the photoemission QE. A thin layer of a material such as diamondoid or DLC have been shown to lower the work function of nanowires. The diamondoid coating is a selfassembled monolayer of molecules whose dipolelike image-charge fields reduce the work function [23]. And, as described in Sec. IV, a few-nanometers-thick layer of DLC allows resonant tunneling from the silicon into the vacuum via the quantum states of the $\mathrm{QW}$ on the tip of the SiNW [24].

\section{METACATHODE ENGINEERING DESIGN}

Figure 13 shows the metacathode design with specifications given in Table III. The drawing shows the design at three length scales spanning 6 orders magnitude from millimeters to nanometers. Beginning at the millimeter scale, a rectangular array of SiNWs extends over an area of a millimeter by a millimeter with $2.5 \times 10^{5} \mathrm{SiNWs} / \mathrm{mm}^{2}$. Assuming each SiNW is an electron emitter, then emission from a 1-mm-diameter area will produce a beam consisting of 200 000 low-charge beamlets which quickly homogenize into a single high-charge electron bunch.

The dimensions of the SiNWs and other design details can be seen in the micron-scale drawing. The doped SiNWs forming the wire array are $1.35 \mu \mathrm{m}$ tall with a separation of $2 \mu \mathrm{m}$. Each SiNW has a radius of $30 \mathrm{~nm}$, and the tip is ellipsoid shaped to give a field enhancement factor of 400 . This makes the tip field $2 \mathrm{GV} / \mathrm{m}$ with an applied field of $5 \mathrm{MV} / \mathrm{m}$. The nanometer-scale drawing shows that the SiNW tip is coated with a 5-nm-thick layer of DLC or a monolayer of diamondoid to reduce the work function.

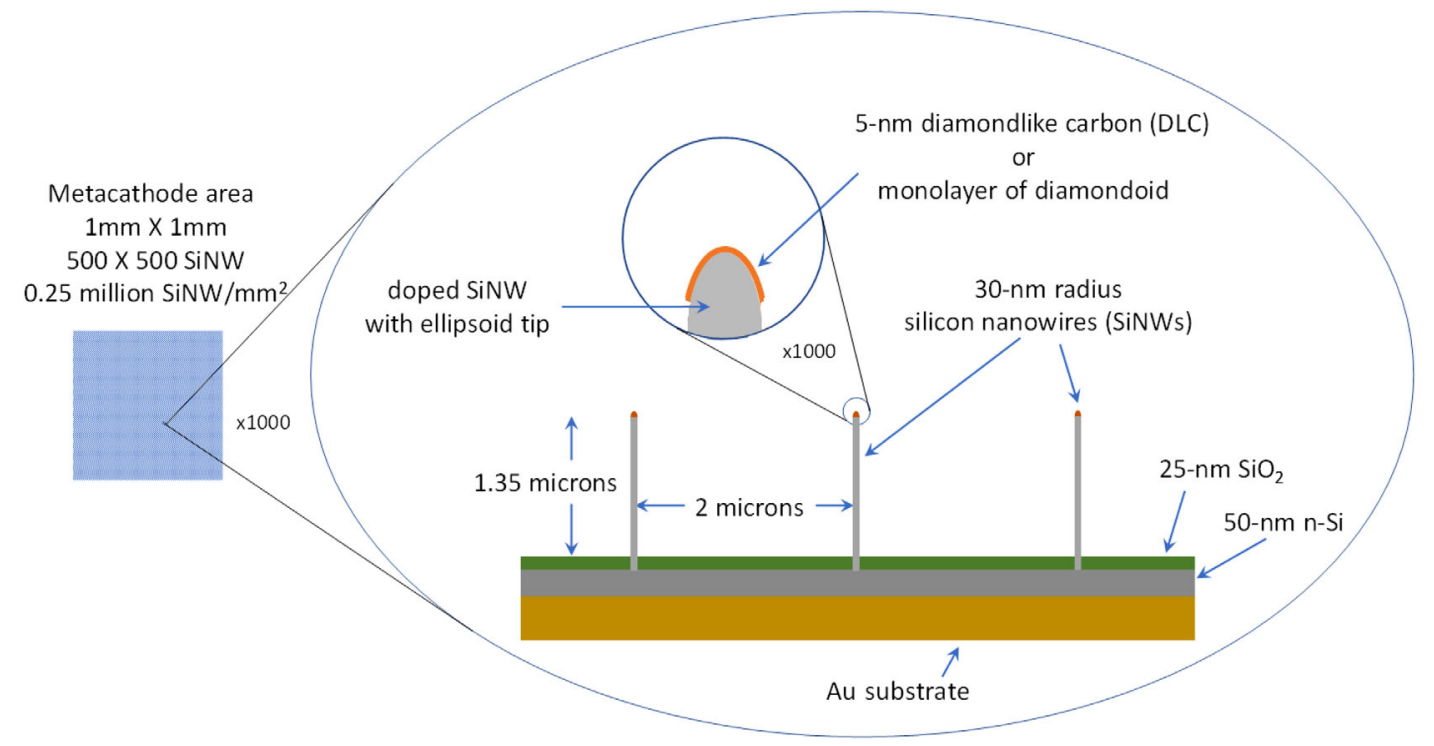

FIG. 13. Conceptual drawing of the metacathode. 
The SiNWs penetrate a top layer of $25-\mathrm{nm} \mathrm{SiO}_{2}$ and terminate in $50 \mathrm{~nm}$ of $n$-type silicon (200- $\Omega \mathrm{m}$ resistance) over a high-conductivity (like gold) substrate. This oxidesilicon-metal substrate design copies that of Björk et al. [19] and is similar to the oxide-metal configuration for a single-photon QW light source [25]. This combination of dielectric and metal substrate is included in the metacathode design, quoting Claudon et al. "to avoid detrimental plasmonic antenna effects at the nanowire-metallic film interface..." [25], which for the metacathode design means the oxide helps to muffle the high-frequency, $\left(\omega>\omega_{t}\right)$ surface plasmon effects and the $n$-type silicon layer conducts current between the nanometer scale of the SiNW to the mesoscale.

\section{CONCLUSIONS AND DISCUSSION}

This paper shows that it is possible to design cathode surfaces which mitigate the space charge limit due to the image charge and make the cathode appear "transparent" to the electrons. The resulting increase in the total accelerating electric field at the cathode's surface can be significant for high-charge bunches, especially in low-voltage guns. As described in Sec. IV C, the SCL of a metacathode with a field of $5 \mathrm{MV} / \mathrm{m}$ was increased a factor of 14 (from $143 \mathrm{pC}$ to $2 \mathrm{nC}$ ) compared to the SCL of a normal cathode. This means the metacathode allows high bunch charge operation in low-voltage electron guns.

The metacathode design presented here is a major departure from standard cathodes, which are intentionally fabricated to be physically and chemically flat. Instead, the wire-array metacathode described in this paper consists of a dense pattern of silicon nanowires with a surface density of $2.5 \times 10^{5} \mathrm{SiNWs} / \mathrm{mm}^{2}$. Each SiNW is $60 \mathrm{~nm}$ in diameter and $1.35 \mu \mathrm{m}$ high above an insulating layer of $\mathrm{SiO}_{2}$ just $25 \mathrm{~nm}$ thick. The $\mathrm{SiNW}$ penetrates the $\mathrm{SiO}_{2}$ layer and electrically connects with 50-nm-thick layer of $n$-type silicon on top of an $\mathrm{Au}$ substrate.

This is truly atom-by-atom engineering. Because a silicon atom has a diameter of $0.111 \mathrm{~nm}$, there are only 540 atoms across the SiNW's diameter of $60 \mathrm{~nm}$. It is this fantastic capability of modern lithography to accurately fabricate large areas of these atom-scale structures which makes metacathodes possible. As a result, the specifications for the metacathode design are shamelessly borrowed from this work. Specifically, the dimensions of a proven vertical SiNW field effect transistor are used in the metacathode design.

While not discussed in this paper, there remain the important topics of beam brightness and emittance of the metacathode. The metacathode intrinsic emittance is related to the energy spread of the QW state through which the emission occurs. And it is expected that most of the metacathode's extrinsic emittance growth occurs during the expansion and merger of the beamlets as they accelerate from the SiNW tips. These and related topics will be revisited in future papers.

However, in anticipation of these future discussions, it is interesting to comment that lower intrinsic emittance should be possible for emission via resonant tunneling. This is a result of the emission proceeding through a single state in the QW which acts like an energy filter on the flux of electrons passing through the QW from the SiNW's conduction band and onto the vacuum. Ideally, the intrinsic emittance could be as small as the energy width of the quantum state. According to the uncertainty principle, the width is related to the inverse of the decay time. Therefore, a fast decay rate (lots of electrons emitted) implies a short decay time leading to a large energy spread of the quantum state. Hence, it appears that, like the QE and emittance of other cathodes, an increase in emission also increases the intrinsic emittance. Future studies should investigate the interplay between the transmission through the barriers, the state's energy spread, and the emission efficiency.

In addition, the effects that intra- and interband transitions have on the metasurface's dielectric function should be investigated. These studies should include topologies such as Dirac cones, Weyl points, and other exotic states. Dirac cones and a band structure in the cathode's dielectric function can be produced using a wire pattern with two or more different-sized SiNWs [26].

This work opens a new direction for electron source research with direct applications in accelerator technology. Metacathodes present the exciting prospect of controlling image- and space charge forces at the cathode surface on the micron scale by custom engineering of the cathode's dielectric function.

\section{ACKNOWLEDGMENTS}

I acknowledge lively and enlightening discussions with H. Padmore and A. Schroeder. And I thank the journal referees for their constructive critiques of the manuscript. Their comments encouraged me to develop a detailed metacathode design. I acknowledge SLAC for providing access to online journals which are behind pay walls. This work was solely funded by the author.

[1] L. Serafini, Short bunch blow-out regime in RF photoinjectors, AIP Conf. Proc. 413, 321 (1997).

[2] O. J. Luiten, S. B. van der Geer, M. J. de Loos, F. B. Kiewiet, and M. J. van der Wiel, How to Realize Uniform Three-Dimensional Ellipsoidal Electron Bunches, Phys. Rev. Lett. 93, 094802 (2004).

[3] P. Musumeci, J. T. Moody, R. J. England, J. B. Rosenzweig, and T. Tran, Experimental Generation and Characterization of Uniformly Filled Ellipsoidal Electron-Beam Distributions, Phys. Rev. Lett. 100, 244801 (2008).

[4] J. David Jackson, Classical Electrodynamics, 3rd ed. (Wiley, New York, 1999), pp. 154-157. 
[5] A. Liebsch, Electronic Excitations at Metal Surfaces (Plenum, New York, 1997), p. 70.

[6] N. W. Ashcroft and N. David Mermin, Solid State Physics (Thompson Learning, Toronto, 1976), pp. 16-20.

[7] C. Ghosh, Photoemission and optical processes in multialkali photocathodes, Phys. Rev. B 22, 1972 (1980).

[8] M. Born and E. Wolf, Principles of Optics, sixth (corrected) ed. (Pergamon, New York, 1986), pp. 624-627.

[9] An Engineering Guide to Photoinjectors, edited by T. Rao and D. H. Dowell, arXiv:1403.7539.

[10] J. W. Brown and R. V. Churchill, Fourier Series and Boundary Value Problems, 6th ed. (McGraw-Hill, New York, 2001), p. 215.

[11] J. D. Jackson, Classical Electrodynamics (Ref. [4]), p. 21.

[12] W. Rotman, Plasma simulation by artificial dielectrics and parallel-plate media, IRE Trans. Antennas Propag. 10, 82 (1962).

[13] J. B. Pendry, A. J. Holden, W. J. Steward, and I. Youngs, Extremely Low Frequency Plasmons in Metallic Mesostructures, Phys. Rev. Lett. 76, 4773 (1996).

[14] W. Cai and V. Shalaev, Optical Metamaterials (Springer, New York, 2010), Sec. IV. 3.

[15] Z. Huang, N. Geyer, P. Werner, J. de Boor, and U. Gosele, Metal-assisted chemical etching of silicon: A review, Adv. Mater. 23, 285 (2010).

[16] Z. Huang, X. Zhang, M. Reiche, L. Liu, W. Lee, T. Shimizu, S. Senz, and U. Gosele, Extended arrays of vertically aligned sub-10 nm diameter Si nanowires by metal-assisted chemical etching, Nano Lett. 8, 3046 (2008).

[17] K. Seo, M. Wober, P. Steinvurzel, E. Schonbrun, Y. Dan, T. Ellenbogen, and K. B. Crozier, Multicolored vertical silicon nanowires, Nano Lett. 11, 1851 (2011).
[18] Z. Huang, H. Fang, and J. Zhu, Fabrication of silicon nanowire arrays with controlled diameter, length, and density, Adv. Mater. 19, 744 (2007).

[19] M. T. Björk, O. Hayden, H. Schmid, H. Riel, and W. Riess, Vertical surround-gated silicon nanowire impact ionization field-effect transistors, Appl. Phys. Lett. 90, 142110 (2007).

[20] S. M. Sze and K. K. Ng, Physics of Semiconductor Devices, 3rd ed. (Wiley, New York, 2007), pp. 104-105.

[21] V. Litovchenko, A. Evtukh, Yu. Kryuchenko, N. Goncharuk, O. Yilmazoglu, K. Mutamba, H. L. Hartnagel, and D. Pavlidis, Quantum-size resonance tunneling in the field emission phenomenon, J. Appl. Phys. 96, 867 (2004).

[22] High Voltage Vacuum Insulation, Basic Concepts and Technological Practice, edited by R. Latham (Academic, New York, 1995).

[23] K. T. Narasimha, C. Ge, J. D. Fabbri, W. Clay, B. A. Tkachenko, A. A. Fokin, P. R. Schreiner, J. E. Dahl, R. M. K. Carlson, Z. X. Shen, and N. A. Melosh, Ultralow effective work function surfaces using diamondoid monolayers, Nat. Nanotechnol. 11, 267 (2016).

[24] S. M. Sze, Physics of Semiconductor Devices (Ref. [20]), pp. 454-459.

[25] J. Claudon, J. Bleuse, N. S. Malik, M. Bazin, P. Jaffrennou, N. Gregersen, C. Sauvan, P. Lalanne, and J.-M. Gerard, A highly efficient single-photon source based on a quantum dot in a photonic nanowire, Nat. Photonics 4, 174 (2010).

[26] S. Yves, T. Berthelot, M. Fink, G. Lerosey, and F. Lemoult, Measuring Dirac Cones in a Subwavelength Metamaterial, Phys. Rev. Lett. 121, 267601 (2018). 